\title{
Intrauterine Growth and its Relationship to Size and Shape at Birth
}

\author{
PETER C. HINDMARSH, MICHAEL P. P. GEARY, CHARLES H. RODECK, JOHN C. P. KINGDOM, \\ AND TIM J. COLE \\ London Centre for Paediatric Endocrinology and Metabolism [P.C.H., M.P.P.G.], Department of Obstetrics \\ and Gynaecology [C.H.R], Centre for Paediatric Epidemiology and Biostatistics [T.J.C.], Institute of Child \\ Health, University College London, UK. Program in Development and Fetal Health [J.C.P.K], Samuel \\ Lunenfeld Research Institute, Mount Sinai Hospital, University of Toronto, Toronto, Canada
}

\section{ABSTRACT}

\begin{abstract}
Birth size and shape are commonly used as indicators of fetal growth. Epidemiologic studies have suggested a relationship between birth size and the risk of developing cardiovascular disease in later life. Certain "growth phenotypes" have been linked to the development of certain components of cardiovascular disease, particularly babies who display disproportional growth in utero. These observations are based on retrospective analysis of historical data sets. If the "Fetal Origins of Adult Disease" hypothesis is to be generalisable to the present day, then it is essential to establish whether these "growth phenotypes" exist within the normal distribution of birth size. The UCL Fetal Growth Study is a prospective study of antenatal fetal growth assessed by ultrasound at 20 and 30 wk gestation in 1650 low risk, singleton, white pregnancies. Measures of birth size were obtained and analyzed by principal components to explain shape at birth. Birth measures were also related to antenatal growth measurements to determine the strength of ultrasound evaluation in determining subsequent growth. There was significant sexual dimorphism in all measures at birth, with males heavier, longer, and leaner than females. From 20 wk of gestation onwards, males had a significantly larger head size than females. Parity, maternal height, and body mass index were important determinants of birth weight $(p<0.001)$. Cigarette smoking influenced birth weight, length, and head circumference $(p<0.001)$ but had no effect on placental size. Principal component analysis revealed
\end{abstract}

that proportionality was the predominant size/shape at birth $(55 \%$ of variance explained). A further $18 \%$ of variance was explained by a contrast between weight, head circumference, and length versus three skinfolds. Anthropometric measures as assessed by ultrasound at 20 and $30 \mathrm{wk}$ gestation were poor predictors of birth length, weight, and head circumference (adjusted $\mathrm{R}^{2} 18,40$, and $28 \%$ at 30 wk gestation scan, respectively). These predictions were not improved by including growth patterns between 20 and 30 wk. There is sexual dimorphism in a number of anthropometric measures at birth and in utero. These sex differences are important determinants of body size and shape. In a low risk population delivering at term, body shape was largely determined by proportionality between anthropometric measures. The low correlations between antenatal measures and birth size suggest that it is unwise to ascribe birth shape phenotypes to adverse events at any particular stage of gestation. The weak relationship also suggests that routine antenatal scans around 30 wk of gestation to predict growth problems are unlikely to be of benefit in the majority of cases. (Pediatr Res 52: 263-268, 2002)

Abbreviations
ANOVA, Analysis of variance
BMI, Body mass index
SDS, SD score

Birth weight is commonly used as an indicator of fetal growth in epidemiologic studies and obstetric practice to assess the effects of pathologic processes and possible therapeutic

Received July 11, 2001; accepted March 25, 2002.

Correspondence and reprint requests: P.C. Hindmarsh, M.D., Cobbold Laboratories, Middlesex Hospital, Mortimer Street, London, W1N 8AA, UK; e-mail: p.hindmarsh@ucl.ac.uk

Study supported by grants from the British Heart Foundation, Children Nationwide UK, and Pharmacia-Upjohn to PCH. JCPK is funded by the Program in Development and Fetal Health, Samuel Lunenfeld Institute, and the Department of Obstetrics and Gynecology, Mount Sinai Hospital, University of Toronto. TJC is funded by the Medical Research Council of Great Britain.

DOI: 10.1023/01.PDR.0000020600.45753.78 interventions $(1,2)$. Birth weight generally reflects longitudinal growth, although the times of peak length velocity and peak weight velocity differ in utero (3). Based upon this observation, it has been suggested that alterations in the patterns of growth at different stages in gestation will lead to different anthropometric phenotypes at birth. Early pregnancy growth failure might be associated with a proportional reduction in length, weight, and head size at birth (2). Late pregnancy growth failure might be associated with a reduction in weight, fat mass, and perhaps length and weight with "sparing" of brain growth as reflected in a normal head circumference (4-6). These observations have developed from clinical observation 
and from ultrasound evaluations of fetal growth over the last $20 \mathrm{y}$ and tend to represent the extremes of clinical practice (7). Whether similar but more subtle changes take place within the general population is unclear. Serial ultrasound studies have not addressed this question (8) as their design has concentrated predominantly on fetal health and placental vascular development, using measures of fetal growth in isolation and ignoring more subtle changes in anthropometry over time.

Several epidemiologic studies have suggested that small babies, or those that have displayed poor intrauterine growth, have increased rates of cardiovascular disease and noninsulin dependent diabetes as adults $(1,9)$. Growth, particularly the distinction between proportional and disproportional growth, has been implicated or associated with different risk factors for the development of cardiovascular disease (10). The hypothesis is that infants with growth restriction dating from early pregnancy and who display proportionate (or symmetrical) growth restriction develop hypertension, whereas infants with mid-trimester growth restriction who display disproportionate (or asymmetrical) growth restriction develop hypertension and syndrome $X(11)$. These observations assume that extrapolations from the extremes of clinical practice are valid and that measurements of fetal growth parameters at any given stage of pregnancy are predictive of future intrauterine growth as well as size and shape at birth. This may not be the case in normal populations $(12,13)$.

There are no prospective data of contemporaneous populations available to substantiate the idea that these "growth phenotypes" exist within the normal distribution of size at birth. As the incidence of cardiovascular disease is not confined to the extremes of birth size, evaluation of intrauterine growth and its impact on birth size and shape becomes important in assessing the strength of the "Fetal Origins of Adult Disease" hypothesis and its applicability to current populations. In 1650 normal pregnancies from the UCL Fetal Growth Study, we measured fetal anthropometry by serial ultrasound examination at 20 and $30 \mathrm{wk}$ of gestation and compared values with anthropometric measures taken at birth.

\section{PATIENTS AND METHODS}

The patients were consecutive mothers booked at the Department of Obstetrics and Gynaecology at University College London Hospitals between April 1996 and July 1997. The study was approved by the Research Ethics Committee of University College London Hospitals, and written informed consent for participation was obtained from the mother for herself at the commencement of the study and for her newborn child after delivery. 1790 mothers fulfilled the entry criteria and $1650(92 \%)$ agreed to participate. They did not differ in terms of clinical or pregnancy outcomes from those who refused. Inclusion criteria were first prenatal visit before $20 \mathrm{wk}$, ultrasound examination demonstrating a structurally normal single fetus, and a white mother. The exclusion criteria were nuchal translucency or evidence of a major malformation in the ultrasound scan $(n=6)$ or maternal steroid use for chronic inflammatory or thrombotic disorders $(n=16)$. Menstrual dates were used to assign gestational age unless the first ultrasound measurement (crown rump length before $12 \mathrm{wk}$, biparietal diameter for 12-20 wk) differed by $>7 \mathrm{~d}$.

At the first prenatal visit (mean gestation $12.9 \mathrm{wk}$, range 6-20), maternal height was measured with a stadiometer (Holtain Limited, Crymych, UK) and recorded to the nearest 0.1 $\mathrm{cm}$. Weight was measured using Seca scales (CMS Weighing Equipment Limited, London, UK) and recorded to the nearest $0.1 \mathrm{~kg}$. Current cigarette consumption was categorized as nonsmoking, $<10,10$ to 20 , or $>20$ cigarettes per day. Socioeconomic status was determined from age at which full-time education was completed; marital status, occupation, and partner's occupation, and social class assignment was made using the classification of the United Kingdom Office of Population Census and Statistics (14).

In addition to the dating ultrasound measurements, additional examinations were conducted at $20 \mathrm{wk}$ (the standard time for structural evaluation of the fetus) and $30 \mathrm{wk}$ gestation. The uptake by mothers was high for both the second trimester $(96 \%)$ and third trimester scans $(84 \%)$, and reasons for not performing the scans were mainly fetal loss or termination of pregnancy. Only 50 mothers refused both scans. Standard fetal biometric measures were made of head circumference, biparietal diameter, abdominal circumference, and femur length. The $20 \mathrm{wk}$ examinations were undertaken by MPPG and clinic ultrasonographers, whereas at $30 \mathrm{wk}$ all examinations were done by MPPG. Measurements were made using an Acuson 128/Xpi ultrasound machine (Mountain View, CA, USA) with a $5 \mathrm{MHz}$ curvilinear transducer. Each measurement was done three times and the mean recorded. The coefficient of variation of the ultrasound measures was $1 \%$ maximum on the $20 \mathrm{wk}$ scan.

Birth weight was measured using electronic self-calibrating scales (Seca, Birmingham, UK), length by Infantometer (Child Growth Foundation, London, UK) and head circumference and mid arm circumference with a metal tape. Triceps, subscapular, and quadriceps skinfold measurements were made with skinfold calipers (Holtain Limited, Crymych, UK). Three separate measurements were taken and the mean recorded. The coefficient of variation of the measurement error for length was $0.15 \%$ based on 10 infants each measured 5 times by 3 observers.

Statistics. All data were explored for the normality of their distribution and log transformed where appropriate. Values for birth weight, length, and head circumference were expressed as SDS using the 1990 British growth reference (15). A threedimensional plot of birth weight, length, and head circumference was inspected to determine the presence of outliers in any of the three dimensions to determine the prevalence of disproportion in the population. A $t$ test was used to determine differences between the sexes in the anthropometric measures made. ANOVA with the Student-Newman-Keuls post hoc test was used to explore the relationship between parity and birth weight. Multiple linear regression analysis was used to predict birth outcome measures from ultrasound estimates of fetal size. As the ultrasound measures were not all done at exactly 20 or $30 \mathrm{wk}$, they were adjusted to the mean age at measurement. Multiple linear regression analysis was also used to identify factors affecting birth size and gestation. 
Principal component analysis was used to summarize the relationship between variables relating to the size and shape of individuals at birth (16). The process combines variables that might reasonably be assumed to contribute to size at birth into a smaller number of other variables that provide almost all the relevant information about the way in which one individual differs from another. The technique identifies weighted averages (or linear contrasts) of the specified variables that explain as much variation across individuals as possible. These linear contrasts are called principal components or eigenvectors, and there are as many of them as there are variables. Each principal component has an eigenvalue that indicates the amount of variation that it explains, and this can be expressed as a percentage of the total variation. The fitting process is sequential. The first eigenvector maximizes the variation explained, while the second and later eigenvectors explain as much as is possible of the remaining variation. Each eigenvector is constrained to be independent of previously fitted eigenvectors. The eigenvectors are defined in terms of the weightings or loadings given to each variable. The signs of the loadings are important: if they are all the same, then the principal component represents a weighted average of the variables, a measure of size. This is usually the first and largest principal component. When some of the loadings are positive and others negative, as happens with the second and later components, the eigenvector represents the difference between those variables with positive sign and those with negative sign.

As an example, if only three factors are chosen, they can be visualized as a three-dimensional plot yielding an ovoid. The first principal component is the distance along the length of the ovoid, the second the distance along the widest direction perpendicular to this, and the third the remaining measurement perpendicular to the other two.

Principal component analysis is based either on the covariance matrix or on the correlation matrix of the chosen variables. Here, the correlation matrix is used to remove the effect of different scales of measurement.

\section{RESULTS}

General. Of the 1650 women who booked, 1484 delivered a live infant, of whom 70 were preterm deliveries and 196 developed complications of pregnancy. Of the 166 who did not complete the study, 127 had moved away or been lost to follow-up, 28 had either a miscarriage or termination of pregnancy, and 11 withdrew. Analysis here is confined to those infants delivered at term free of pregnancy complications $(n=$ 1218 or $82 \%$ of those delivered). The clinical and anthropometric details of the women are shown in Table 1. Anthropometric measures were no different from the UK population. The social class distribution was similar to that of the UK with a slight preponderance of social class II (I, 13\%; II, 41\%; III, $21 \%$; IV, $12 \%$; V , 11\%). $71 \%$ of the cohort were nonsmokers, $9 \%$ were exsmokers, $10 \%$ smoked less than 10 per day, and $11 \%$ smoked 10 or more per day. Although enquiry was made into alcohol consumption, the number admitting to alcohol exposure during pregnancy was too small for further analysis.
Table 1. Clinical and anthropometric details of the UCL cohort of 1218 mothers at booking

\begin{tabular}{lc}
\hline Age $(\mathrm{yr})$ & $31.0(5.6)$ \\
Height $(\mathrm{cm})$ & $164.5(6.8)$ \\
Weight $(\mathrm{kg})$ & $63.6(11.1)$ \\
Body mass index $\left(\mathrm{kg} / \mathrm{m}^{2}\right)$ & $23.6(4.2)$ \\
Parity $(\%)$ & \\
0 & 49 \\
1 & 32 \\
2 & 11 \\
$3+$ & 8 \\
Gestation at booking (wk) & $12.9(2.6)$ \\
Gestation at delivery (wk) & $39.7(1.3)$ \\
\hline
\end{tabular}

Data shown as mean with SD in parenthesis.

Intrauterine growth. Table 2 shows the anthropometric measures derived from ultrasound examination at 20 and $30 \mathrm{wk}$ of gestation. There were significant sex differences in abdominal circumference, biparietal diameter, and head circumference, but not femur length.

Size at birth. Details of the anthropometric measures at birth in the 1218 babies born at term following an uncomplicated pregnancy are detailed in Table 3. Birth weight SDS (0.06 SD $0.98)$ and head circumference SDS ( -0.06 SD 1.1) were not significantly different from the UK reference values, and length SDS (-0.13 SD 1.2) only marginally so, indicating that this population was representative, in growth terms, of the UK population (15). Mean placental weight was $672 \mathrm{~g}$ (SD 129). There were significant sex differences in birth weight, length, and head circumference with males heavier, longer, leaner (greater mid-arm circumference but smaller skinfolds), and having larger heads than females.

Table 4 details factors influencing birth size. Birth weight expressed in grams was influenced by sex (see above) and smoking. Cigarette smoking in pregnancy was associated with a reduction in birth weight of $190 \mathrm{~g}$. This effect did not depend on the number of cigarettes smoked, although few mothers smoked more than 20 per day. Of maternal factors influencing birth weight parity, height and BMI at recruitment were im-

Table 2. Anthropometric measures derived from ultrasound examination at 20 and 30 weeks gestation

\begin{tabular}{cccc}
\hline & Male & Female & $p$ \\
\hline Age at measurement & & & \\
20 weeks (750M:705F) & $20.3(1.0)$ & $20.2(1.0)$ & 0.5 \\
30 weeks (682M:652F) & $32.2(1.2)$ & $32.3(1.3)$ & 0.04 \\
Abdominal circumference (mm) & & & \\
20 weeks (748M:702F) & $157.8(13.3)$ & $155.2(12.7)$ & $<0.001$ \\
30 weeks (682M:652F) & $288.5(17.5)$ & $285.7(17.9)$ & 0.001 \\
Biparietal diameter (mm) & & & \\
20 weeks (748M:704F) & $50.1(3.5)$ & $48.9(3.4)$ & $<0.001$ \\
30 weeks (604M:568F) & $85.2(4.0)$ & $83.9(4.2)$ & $<0.001$ \\
Head circumference (mm) & & & \\
20 weeks (749M:704F) & $179.7(12.3)$ & $176.0(11.7)$ & $<0.001$ \\
30 weeks (604M:568F) & $302.6(13.3)$ & $297.8(14.3)$ & $<0.001$ \\
Femur length (mm) & & & \\
20 weeks (739M:690F) & $32.7(3.0)$ & $32.6(2.7)$ & 0.3 \\
30 weeks (681M:652F) & $62.1(3.1)$ & $62.1(3.2)$ & 0.8 \\
\hline
\end{tabular}

Data shown as mean with SD in parenthesis. Results adjusted to mean age at measurement. 
Table 3. Anthropometric measures at birth in 1218 term infants from uncomplicated pregnancies

\begin{tabular}{lccc}
\hline & Male & Female & \\
& $(n=573-632)$ & $(n=521-586)$ & $p$ \\
\hline Weight (kg) & $3.52(0.49)$ & $3.39(0.44)$ & $<0.001$ \\
$\quad$ Weight SDS & $0.05(0.98)$ & $0.11(0.97)$ & 0.30 \\
Length (cm) & $50.5(2.5)$ & $49.7(2.2)$ & $<0.001$ \\
Length SDS & $-0.15(1.17)$ & $-0.12(1.13)$ & 0.70 \\
Head circumference (cm) & $34.9(1.5)$ & $34.3(1.4)$ & $<0.001$ \\
Head circumference SDS & $-0.10(1.13)$ & $0.00(1.09)$ & 0.10 \\
Mid-arm circumference (cm) & $10.6(1.02)$ & $10.4(1.00)$ & 0.02 \\
Triceps skinfold (mm) & $5.78(1.60)$ & $5.86(1.50)$ & 0.3 \\
Subscapular skinfold (mm) & $5.28(1.55)$ & $5.48(1.60)$ & 0.03 \\
Quadriceps skinfold (mm) & $7.24(1.90)$ & $7.62(1.87)$ & 0.001 \\
Placental weight (g) & $677(131)$ & $666(127)$ & 0.17 \\
\hline
\end{tabular}

Data shown as mean with SD in parenthesis.

Table 4. Multiple regression analysis of factors affecting birth size and gestation

\begin{tabular}{|c|c|c|c|c|}
\hline \multirow{2}{*}{$\frac{\text { Covariate }}{\text { Birth weight }(\mathrm{g})(n=1129)}$} & \multicolumn{3}{|c|}{ Coefficient t-ratio Adjusted $\mathrm{R}^{2}$} & \multirow[t]{2}{*}{$p$} \\
\hline & & & & \\
\hline Female sex & -145 & -6.0 & 26.0 & $<0.001$ \\
\hline Gestation & 124 & 13.1 & & $<0.001$ \\
\hline Smoking in pregnancy & -189 & -6.1 & & $<0.001$ \\
\hline Primiparity & -184 & -7.6 & & $<0.001$ \\
\hline Maternal height $(\mathrm{cm})$ & 16 & 8.8 & & $<0.001$ \\
\hline Maternal body mass index $\left(\mathrm{kg} / \mathrm{m}^{2}\right)$ & 21 & 7.0 & & $<0.001$ \\
\hline \multicolumn{5}{|l|}{ Birth length $(\mathrm{cm})(n=1102)$} \\
\hline Female sex & -0.72 & -5.7 & 19.5 & $<0.001$ \\
\hline Gestation & 0.59 & 11.9 & & $<0.001$ \\
\hline Smoking in pregnancy & -0.65 & -4.0 & & $<0.001$ \\
\hline Primiparity & -0.59 & -4.5 & & $<0.001$ \\
\hline Maternal height $(\mathrm{cm})$ & 0.074 & 7.6 & & $<0.001$ \\
\hline Maternal body mass index $\left(\mathrm{kg} / \mathrm{m}^{2}\right)$ & 0.055 & 3.5 & & $<0.001$ \\
\hline \multicolumn{5}{|l|}{ Head circumference $(\mathrm{cm})(n=1107)$} \\
\hline Female sex & -0.56 & -7.2 & 19.3 & $<0.001$ \\
\hline Gestation & 0.34 & 10.9 & & $<0.001$ \\
\hline Smoking in pregnancy & -0.44 & -4.4 & & $<0.001$ \\
\hline Primiparity & -0.33 & -4.1 & & $<0.001$ \\
\hline Maternal height $(\mathrm{cm})$ & 0.038 & 6.3 & & $<0.001$ \\
\hline Maternal body mass index $\left(\mathrm{kg} / \mathrm{m}^{2}\right)$ & 0.056 & 5.8 & & $<0.001$ \\
\hline \multicolumn{5}{|l|}{ Placental weight $(\mathrm{g})(n=987)$} \\
\hline Female sex & -15.8 & -2.0 & 6.7 & 0.05 \\
\hline Gestation & 14.3 & 4.6 & & $<0.001$ \\
\hline Smoking in pregnancy & -1.8 & -0.2 & & 0.8 \\
\hline Primiparity & -30 & -3.7 & & $<0.001$ \\
\hline Maternal height $(\mathrm{cm})$ & 3.6 & 5.9 & & $<0.001$ \\
\hline Maternal body mass index $\left(\mathrm{kg} / \mathrm{m}^{2}\right)$ & 3.6 & 3.8 & & $<0.001$ \\
\hline \multicolumn{5}{|l|}{ Gestation (weeks) $(n=1129)$} \\
\hline Female sex & -0.04 & -0.5 & 0.8 & 0.6 \\
\hline Smoking in pregnancy & -0.12 & -1.2 & & 0.2 \\
\hline Primiparity & 0.23 & 3.0 & & 0.003 \\
\hline Maternal height $(\mathrm{cm})$ & 0.003 & 0.5 & & 0.6 \\
\hline Maternal body mass index $\left(\mathrm{kg} / \mathrm{m}^{2}\right)$ & -0.01 & -1.1 & & 0.3 \\
\hline
\end{tabular}

portant factors. Maternal age did not influence birth weight. Similar effects were observed with birth length and head circumference.

Placental weight was slightly lower in females by approximately $16 \mathrm{~g}$ (2.4\% reduction on male placental weight) despite a $4 \%$ reduction on male birth weight. Smoking was not associated with any change in placental weight despite a $190 \mathrm{~g}$ reduction in birth weight.

Gestation was not influenced by any of the factors other than being slightly longer in primiparous women.
The relationship between birth weight, length, and head circumference was explored with three-dimensional plots on a computer screen. The plots and the rotation of the plots did not reveal any significant deviation from the predicted smooth ovals expected if proportionality was the dominant shape pattern at birth. The contribution of the different anthropometric measures to overall shape at birth was analyzed by principal components as shown in Table 5. The first eigenvector, explaining $55 \%$ of the variance, has loadings that are all of the same sign and of similar magnitude, indicating a measure of body size. The second eigenvector, explaining $18 \%$ of the variance, is a contrast between size, represented by birth weight, length, and head circumference, and fat represented by the three skinfolds. The third eigenvector compares length with midarm circumference, and the fourth compares head with length. Overall, $73 \%$ of variance in shape at birth is explained by the first two eigenvectors and $88 \%$ by the first four (Table 5).

Prediction of birth size from ultrasound. Anthropometric measures as assessed by ultrasound at both 20 and $30 \mathrm{wk}$ of gestation were poor predictors of birth weight, length, and head circumference SDS (Table 6). The 30 wk ultrasound was a better predictor than at $20 \mathrm{wk}$, with an adjusted $\mathrm{R}^{2}$ for birth weight, length, and head circumference SDS of 40, 18, and $28 \%$, respectively. The ultrasound prediction was not improved by adding changes in anthropometric measures between 20 and 30 wk (adjusted $\mathrm{R}^{2}$ for birth weight, length, and head circumference SDS, 41,19 , and $29 \%$, respectively).

\section{DISCUSSION}

These data demonstrate that the major determinant of shape at birth is proportionality between the anthropometric measures of size at birth, and that size (made up of weight, length, and head circumference) opposing body fatness is an important additional determinant. The two factors account for $73 \%$ of shape at birth. It is not possible from the data to ascribe a significant contribution to birth shape made by classical concepts of disproportionate growth. Disproportional growth, for example the preservation of head growth at the expense of total body weight gain, certainly exists and has been well documented in the pediatric and obstetric literature (4-6). The observations accord with knowledge of the different growth patterns of body weight and length as well as individual organ

Table 5. Principal component analysis of factors contributing to size and shape at birth in 1092 term uncomplicated pregnancies

\begin{tabular}{lcrrr}
\hline \multirow{2}{*}{\multicolumn{1}{c}{ Factors }} & \multicolumn{4}{c}{ Eigenvectors } \\
\cline { 2 - 5 } & $\mathrm{V} 1$ & $\mathrm{~V} 2$ & \multicolumn{1}{c}{ V3 } & \multicolumn{1}{c}{ V4 } \\
\hline Birth weight & $\mathbf{0 . 4 4}$ & -0.27 & -0.09 & -0.18 \\
Length & $\mathbf{0 . 3 4}$ & $\mathbf{- 0 . 4 4}$ & $\mathbf{0 . 5 6}$ & $\mathbf{- 0 . 4 8}$ \\
Head circumference & $\mathbf{0 . 3 2}$ & -0.48 & 0.02 & $\mathbf{0 . 7 9}$ \\
Mid-arm circumference & $\mathbf{0 . 3 9}$ & -0.08 & $\mathbf{- 0 . 7 8}$ & -0.25 \\
Triceps skinfolds & $\mathbf{0 . 3 7}$ & 0.42 & 0.21 & 0.15 \\
Subscapular skinfolds & $\mathbf{0 . 3 7}$ & 0.47 & 0.14 & 0.15 \\
Quadriceps skinfolds & $\mathbf{0 . 4 1}$ & 0.30 & 0.05 & -0.08 \\
Eigenvalue & $\mathbf{3 . 8 8}$ & $\mathbf{1 . 2 8}$ & $\mathbf{0 . 5 5}$ & $\mathbf{0 . 4 5}$ \\
Variance proportion $(\%)$ & $\mathbf{5 5}$ & $\mathbf{1 8}$ & $\mathbf{8}$ & $\mathbf{7}$ \\
\hline
\end{tabular}

Figures in bold show strongest contrasts. 
Table 6. Fetal ultrasonography at 20 and 30 wk gestation as predictors of size at birth

\begin{tabular}{|c|c|c|c|c|}
\hline Measure & Coefficient & t-ratio & $p$ & $\mathrm{R}^{2}$ \\
\hline \multicolumn{5}{|l|}{ Ultrasound $20 \mathrm{wk}$} \\
\hline Birth weight SDS $(n=1167)$ & & & & 11.6 \\
\hline Abdominal circumference (mm) & 0.019 & 5.9 & $<0.001$ & \\
\hline Femur length $(\mathrm{mm})$ & 0.049 & 2.7 & 0.006 & \\
\hline Birth length SDS $(n=1139)$ & & & & 5.3 \\
\hline Abdominal circumference (mm) & 0.012 & 3.1 & 0.002 & \\
\hline Femur length (mm) & 0.066 & 3.6 & $<0.001$ & \\
\hline Head circumference SDS $(n=1166)$ & & & & 8.7 \\
\hline Head circumference $(\mathrm{mm})$ & 0.025 & 6.8 & $<0.001$ & \\
\hline \multicolumn{5}{|l|}{ Ultrasound $30 \mathrm{wk}$} \\
\hline Birth weight SDS $(n=951)$ & & & & 39.7 \\
\hline Abdominal circumference (mm) & 0.031 & 14.6 & $<0.001$ & \\
\hline Femur length $(\mathrm{mm})$ & 0.052 & 4.5 & $<0.001$ & \\
\hline Biparietal diameter (mm) & 0.035 & 4.2 & $<0.001$ & \\
\hline Birth length SDS $(n=932)$ & & & & 17.6 \\
\hline Abdominal circumference (mm) & 0.020 & 6.6 & $<0.001$ & \\
\hline Femur length $(\mathrm{mm})$ & 0.077 & 4.8 & $<0.001$ & \\
\hline Head circumference $(\mathrm{mm})$ & 0.013 & 3.7 & $<0.001$ & \\
\hline Head circumference SDS $(n=935)$ & & & & 27.8 \\
\hline Abdominal circumference (mm) & 0.015 & 5.7 & $<0.001$ & \\
\hline Head circumference $(\mathrm{mm})$ & 0.023 & 5.5 & $<0.001$ & \\
\hline Biparietal diameter (mm) & 0.049 & 3.5 & $<0.001$ & \\
\hline
\end{tabular}

Results adjusted for age at ultrasound measurement.

growth $(3,17)$. However, the validation of these observations has stemmed from fetal ultrasonography where the criterion for sequential evaluation was a reduction in fetal head growth and where a larger reduction in the anthropometric measures was subsequently noted. Although eigenvector 2 looks to be a measure of "disproportion" it should be remembered that the anthropometric "stand-off" it describes between size and fatness is after adjustment had been made for eigenvector 1 , size. This means that this "growth phenotype" operates independently of birth size, particularly birth weight. The disproportion that has been identified in clinical situations and implicated in epidemiologic studies includes a birth weight component that is not present in this low risk population.

Interpreting the eigenvectors derived from principal component analysis is inevitably subjective, but the analysis does provide an unbiased estimate of the contributions made by a number of biologically plausible factors to shape at birth. The method also avoids preconceived ideas as to what constitutes disproportional growth and what cut-off values represent extremes of size. This is particularly important when the influence of parity and other maternal factors on birth weight is considered, e.g. the increase of $184 \mathrm{~g}$ from para 0 to para $1+$ will alter the definition of low birth weight.

Cigarette smoking was an important environmental factor influencing birth weight, which confirmed previous reports (18). Regression analysis revealed that birth weight, length, and head circumference were reduced by cigarette smoking, whereas fat measures were unaffected. Placental weight was unaffected in this population, in contrast to the findings of Williams et al. (19) but consistent with those of Howe et al. (20). The differences may be explained, at least partly, by the unbiased selection of patients in our cohort and the small proportion of heavy smokers. The reduction in birth weight was not associated with fat changes, and the concurrent reduc- tion in head circumference suggests that the reduction in brain growth is the most important effect of smoking on the fetus.

In addition to this environmental factor, other maternal factors influencing birth size were also noted. The effect of maternal height and BMI at booking were confirmed, indicating that this low risk population does not appear to behave in a manner different from that reported in the obstetric literature. We were unable to detect an effect of sex on gestational age at delivery in this population, which probably reflects the larger sample sizes needed to unmask this effect $(21,22)$

Proportional growth appeared to be the major component of shape at birth in this low risk term population. Shape at birth was also influenced by the sex of the child, with males having greater birth weight, length, and head circumference and smaller skinfolds than females, components similar to eigenvector 2. Despite the differences in birth weight, males were generally leaner than females. These observations are akin to the situation in puberty where males develop a greater muscle mass with less fat accumulation than females (23). This suggests that the appearances at birth also reflect differences in the sex steroid environment between the sexes, although the influence of other metabolic factors, e.g. IGF-1, cannot be excluded.

The sex difference in anthropometric measures was observed in utero as well with a sexually dimorphic pattern in head size observable at $20 \mathrm{wk}$ gestation. This effect appeared to develop between 10 and 20 wk gestation as it was absent at the booking scan (mean (SD) biparietal diameter, male, 24.0 (SD 4.3) mm; female, 23.7 (SD 4.4) mm). Abdominal circumference was greater in the males, whereas there was no difference in femur length. The former might well represent differences in maturational rates of abdominal structures such as the liver. Femur length difference might not be expected much before $30 \mathrm{wk}$ of gestation given the timing of peak length velocity in utero. However, the mean difference in length at term between the sexes amounts to $0.8 \mathrm{~cm}$, and it is possible to underestimate the sex difference in femur length due to measurement error.

Intrauterine growth has been depicted graphically as a smooth continuous process. The majority of the studies from which the "standards" have been constructed have been crosssectional in nature and can be criticized for poor study design and analysis $(24,25)$. It has largely been assumed that the fetus maintains its own growth channel so that deviation signals an abnormal growth process. The correlation of our measures at 20 and 30 wk gestation with birth measures was generally poor, although the 30 -week values were better. The predictive value of the assessment of size at birth was poor with only the prediction of birth weight at $30 \mathrm{wk}$ gestation performing well $\left(\mathrm{R}^{2}, 40 \%\right)$. This suggests that routine fetal biometry by ultrasound during pregnancy in a low risk population is of little predictive value $(8,26)$. In the first year of life, movement across the centiles takes place as the infants realign their growth trajectory toward their genetically determined stature. As a result, low correlations between successive anthropometric measures in the first year of life are expected. This appears to be the situation in utero as well. Whether or not it is part of the postnatal process or a reflection of variation in other fetal growth regulators, e.g. nutrient supply, remains unclear. 
These data demonstrate that there is sexual dimorphism in a number of anthropometric measures at birth and that the differences are established early during fetal life. The pattern is strongly reminiscent of the effects of sex steroids on body composition during puberty. Differences in body size need to be considered not only at birth but also in the interpretation of intrauterine growth. In a low risk population delivering at term, body shape was largely determined by proportionality between anthropometric measures, with the sex differences outlined above an additional factor. Classical disproportion was uncommon. The poor correlation between body size measurements particularly early in pregnancy and size at birth implies that it would be unwise to ascribe birth shape "phenotypes" to adverse events at particular stages of gestation in low risk pregnancies. It is possible that our restricting the sample to term infants free of pregnancy complications $(82 \%$ of the unrestricted sample) has generated this phenomenon. We have attempted to exclude many of the potentially confounding variables that influence shape and size to best describe the relationship in the low risk population. This does not imply that adverse events in pregnancy might affect shape size relations in preterm infants or other situations. We chose this low risk population deliberately as one of the aims of the study was to delineate whether the phenotypes described by Barker et al. $(10,11)$ were relevant. This is important because the associations put forward by Barker et al. between fetal shape/size and adult disease have to operate across the low risk population to be consistent with known cardiovascular epidemiology. Furthermore, this poor relationship suggests that routine assessment of fetal size in mid or late gestation is unhelpful in trying to predict individuals liable to suffer from intrauterine growth restriction. Finally, the observations surrounding parity and birth size together with the above observations suggest that further developments of intrauterine growth reference charts need to be undertaken to include a longitudinal component and to contain adjustments for maternal parity and size.

Acknowledgments. We thank the midwifery staff of University College Hospitals for their assistance in the creation of the Fetal Growth Study cohort and Research Midwives Marcia Persaud and Jean Wilshin for coordinating the Study.

\section{REFERENCES}

1. Frankel S, Elwood P, Sweetnam P, Yarnell J, Davey Smith G 1996 Birthwight, body mass index in middle age and incident coronary heart disease. Lancet 348:1478-1480

2. Smith GCS, Smith MFS, McNay MB, Fleming JEE 1998 First-trimester growth and the risk of low birth weight. N Engl J Med 339:1817-1822

3. Tanner JM 1989 Foetus into Man, 2nd Ed. Castlemead Publications, Ware, Herts

4. Urrusti J, Yoshida P, Velasco L, Frenk S, Rosado A, Sosa A, Morales M, Yoshida T, Metcoff J 1972 Human fetal growth retardation. I. Clinical features of samples with intrauterine growth retardation. Pediatrics 50:547-558

5. Villar J, Belizan JM 1982 The timing factor in the pathophysiology of the intrauterine growth retardation syndrome. Obstet Gynecol Surv 37:499-506

6. Kramer MS, Olivier M, McLean FH, Willis DM, Usher RH 1990 Impact of intrauterine growth retardation and body proportionality on fetal and neonatal outcome. Pediatrics 86:707-713

7. Kingdom JCP, and Smith G Diagnosis and management of IUGR 2000. In: Kingdom JCP and Baker P (eds) Intrauterine Growth Restriction-Aetiology and Management. Springer, London, pp 257-270

8. Bricker L, Neilson JP 2000 Routine ultrasound in late pregnancy (after 24 weeks gestation). Cochrane Review. In: The Cochrane Library, Issue 1. Update Software, Oxford

9. Ravelli AC, van der Meulen JH, Michels RP, Osmond C, Barker DJ, Hales CN, Bleker OP 1998 Glucose tolerance in adults after prenatal exposure to famine. Lancet 351:173-177

10. Barker DJP, Gluckman PD, Godfrey KM, Harding JE, Owens JA, Robinson JS 1993 Fetal nutrition and cardiovascular disease in adult life. Lancet 341:938-941

11. Barker DJP, Hales CN, Fall CHD, Osmond C, Phipps K, Clark PMS 1993 Type 2 (non-insulin dependent) diabetes mellitus, hypertension and hyperlipidaemia (Syndrome X): relation to reduced fetal growth. Diabetologia 36:62-67

12. Seeds JW 1984 Impaired fetal growth. Obstet Gynecol 64:577-584

13. Neilson JP, Munjanja SP, Whitfield CR 1984 Screening for small for dates fetuses: a controlled trial. BMJ 289:1179-1182

14. Office of Federal Statistical Policy and Standards 1991 Standard Occupational Classification, Volume 3. U.S. Government Printing Office, Washington DC

15. Freeman JV, Cole TJ, Chinn S, Jones PRM, White EM, Preece MA 1995 Crosssectional stature and weight reference curves for the UK, 1990. Arch Dis Child $73: 17-24$

16. Armitage P, Berry G 1994 Statistical Methods in Medical Research, 3rd Ed. Blackwell Science, Oxford

17. Gruenwald P 1978 Intrauterine growth. In: Stuve U (ed) Perinatal Physiology. Plenum, New York, pp 1-18

18. Butler NR, Goldstein H, Ross EM 1972 Cigarette smoking in pregnancy: its influence on birth weight and perinatal mortality. BMJ 2:127-130

19. Williams LA, Evans SF, Newnham JP 1997 Prospective cohort study of factors influencing the relative weights of the placenta and newborn infant. BMJ 314:18641868

20. Howe DT, Wheeler T, Osmond C 1995 The influence of maternal haemoglobin and ferritin on mid-pregnancy placental volume. Br J Obstet Gynaecol 102:213-219

21. De Zegher F, Devlieger K, Eeckels R 1999 Fetal growth: boys before girls. Horm Res 51:258-259

22. Loos RJF, Derom C, Eeckels R, Derom R, Vlietnick R 2001 Length of gestation and birthweight in dizygotic twins. Lancet 358:560-561

23. Tanner JM 1962 Growth at Adolescence. Blackwell Scientific Publications, Oxford

24. Mahadevan N, Pearce M, Steer P 1994 The proper measure of intrauterine growth retardation is function, not size. Br J Obstet Gynaecol 101:1032-1035

25. Chitty LS, Altman DG, Henderson A, Campbell S 1994 Charts of fetal size: 2 Head Measurements. Br J Obstet Gynaecol 101:35-43

26. Smith GC, Smith MF, MacNay MB, Fleming JE 1997 The relation between fetal abdominal circumference and birthweight: findings in 3512 pregnancies. Br J Obstet Gynaecol 104:186-190 